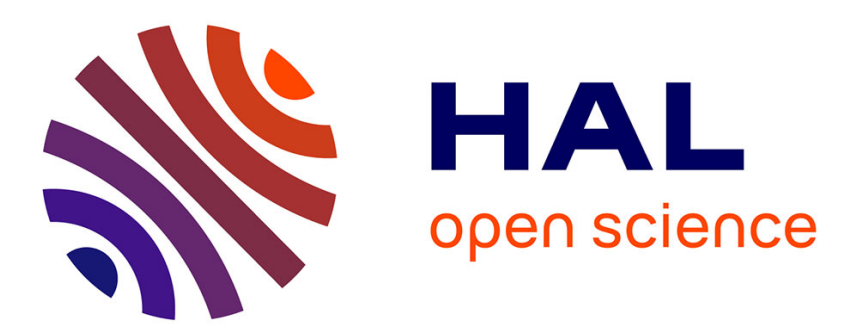

\title{
Resonant vibration of a thin polymer film under optical excitation
}

\author{
Janine Emile, O. Emile, P. Gaudriault
}

\section{To cite this version:}

Janine Emile, O. Emile, P. Gaudriault. Resonant vibration of a thin polymer film under optical excitation. Soft Matter, 2021, 17 (14), pp.3923-3928. 10.1039/d1sm00016k . hal-03225419

HAL Id: hal-03225419

https://hal.science/hal-03225419

Submitted on 20 May 2021

HAL is a multi-disciplinary open access archive for the deposit and dissemination of scientific research documents, whether they are published or not. The documents may come from teaching and research institutions in France or abroad, or from public or private research centers.
L'archive ouverte pluridisciplinaire HAL, est destinée au dépôt et à la diffusion de documents scientifiques de niveau recherche, publiés ou non, émanant des établissements d'enseignement et de recherche français ou étrangers, des laboratoires publics ou privés. 


\title{
Resonant vibration of a thin polymer film under optical excitation.
}

\author{
Janine EMILE, ${ }^{1}$ Olivier EMILE, ${ }^{2}$ and Pierre GAUDRIAULT ${ }^{3}$ \\ ${ }^{1}$ IPR, UMR CNRS 6251, Université Rennes 1, 35042 Rennes cedex, France. \\ ${ }^{2}$ Université de Rennes I, 35042 Rennes Cedex, France.* \\ ${ }^{3}$ Cherry Biotech SAS, Rennes, France.
}

\begin{abstract}
We report on the mechanical excitation of a $220 \mu \mathrm{m}$ thick thermoplastic film in its amorphous state by the radiation pressure of light. By modulating a low power visible laser (from 100 to $600 \mathrm{~mW}$ ) at low frequencies (below $100 \mathrm{~Hz}$ ), we observe a deformation of the film interfaces. The phenomenon, that is independent of the laser wavelength, is amplified at a resonant frequency and reaches $0.68 \mu \mathrm{m}$. The deformation is reversible and varies linearly with the optical power. Using the damped oscillator model, we show that the resonant frequency depends on the surface tension of the film. The associated free energy is then compared with the energy lost, taking into account the contribution of the damping corresponding to the imaginary part of the Young's modulus.
\end{abstract}




\section{INTRODUCTION}

Resonant phenomena are universal in nature. They occur when the frequency of a periodically applied force is in harmonic proportion to a natural frequency of the system on which it acts. They have been extensively considered and exploited in many scientific areas $[1,2]$. Pushing a person on a swing is probably the most common example of it. Among all these resonant phenomena, the vibration of thin films such as membranes bears a special status because of its close links with acoustical vibration [3] and hearing [4]. It is thus compulsory to fully characterize the mechanical properties of the membranes [5]. This also includes a freely suspended soap film that was early recognized as an archetype of a vibrating membrane [6]. In this case, the vibrating mode is an asymmetric mode, where both interfaces are curved in an asymmetric way.

Recently, the vibration of a soap film in a symmetric mode, where the interfaces of the soap film undulate in anti-phase, has been reported $[7,8]$ at low frequencies. This has also been evidenced in an amorphous material deposited on a glass substrate under optical radiation pressure excitation [9]. The phenomenon has been attributed to the contribution of the surface tensions of the different interfaces probed. Nevertheless, no link has been established with the mechanical properties of the material since: (i) the soap film is very fragile with a limited lifetime due to environmental conditions; (ii) the deposited films under resonant vibrations delaminate from the substrate or deteriorate over time. For these two examples, the duration of experiments with reproducibility was rather short to extract reliable information. One may then wonder whether such resonances could be encountered in polymer films with well-known elastic properties. The aim of this article is thus to investigate the dynamical response of a thin thermoplastic sample excited by modulated light, coupling optical radiation pressure to mechanical resonance phenomena. 


\section{EXPERIMENTAL SET-UP}

We have studied the vibration of a rectangular $60 \times 42 \mathrm{~mm}^{2}$ (thickness $e=220$ $\mu \mathrm{m}$, mass $m=565 \mathrm{mg}$ ) commercially available Topas@8007 film. Topas (abbreviation of Thermoplastic Olefin Polymer of Amorphous Structure) is a Cyclic Olefin Copolymer (COC) [10] that is used for packing, optics and fiber spinning. It is also widely used in the healthcare area because of its biocompatible properties and also in microfluidics [11]. Thin films made of COC can be obtained, with high optical surface quality and with less aging problems [12]. The Topas glass temperature transition is $T_{G}=78^{\circ} \mathrm{C}$, with a Young's modulus $E=2.4 \mathrm{GPa}$ and Poisson's ratio $\sigma=0.41$. Its optical index in the visible spectrum is $n=1.53[13,14]$. In contrast to elastomers where capillary forces induce significant interfacial deformations [15], the surface stress in thermoplastic films is negligible.

Our experimental set up is displayed in figure 1a. A laser beam combiner (L4Cc Oxxius, wavelength $\lambda=532 \mathrm{~nm}$, output power $P=250 \mathrm{~mW}$ at the end of the output coupling fiber) is directed on the film nearly at normal incidence. The output power can be increased up to $P=600 \mathrm{~mW}$ by using other wavelengths $(\lambda=404 \mathrm{~nm}$ and $\lambda=488 \mathrm{~nm})$. Because of the optical index difference between the air and the film, there is discontinuity of the linear momentum at the interface $[16,17]$ that induces a force at the interface between the two media. It goes from the higher optical index media towards the lower optical index media. Since the Topas index is $n=1.53$ and the index of the air is around 1 , the force is directed from the Topas to the air, whatever the interface, in particular, for a fixed direction it changes sign from one interface to the other (see figure 1b). More precisely, the force of the transmitted light on the first air/film interface is

$$
\vec{F}_{1}^{t}=(1-n) \frac{4 n}{(n+1)^{2}} N \hbar \vec{k}=(1-n) \frac{4 n}{(n+1)^{2}} \frac{P}{c} \vec{u}
$$

where $N=P / h \nu_{l}$ is the number of photons impinging on the interface per second, $\hbar \vec{k}$ is the linear momentum per photon, $\hbar=h / 2 \pi$ is the reduced Planck constant, 


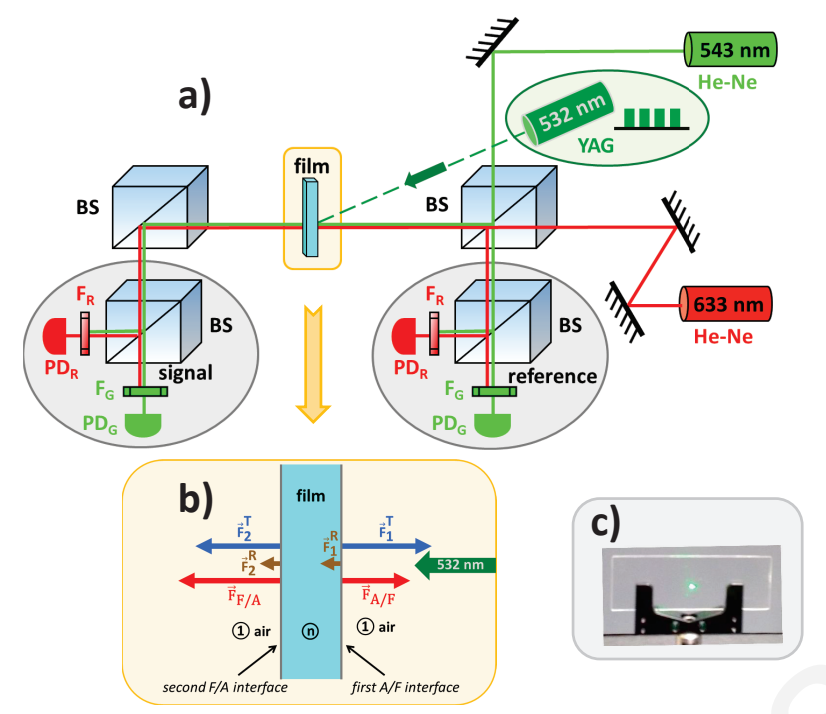

FIG. 1. a) Experimental set up: a $532 \mathrm{~nm}$ intensity modulated laser excites a polymer film. Its thickness variation is measured with the interference fringes of two He-Ne lasers $\left(\lambda_{R}=633 \mathrm{~nm}\right.$ and $\left.\lambda_{G}=543 \mathrm{~nm}\right)$ detected on photodiodes $\mathrm{PD}_{\mathrm{R}}$ and $\mathrm{PD}_{\mathrm{G}}$. Reference signals correct variations of the laser intensity. F: color filters. BS: beam splitter. The temperature is controlled to be $20 \pm 0.2^{\circ} \mathrm{C}$. b) Higher magnification of the film showing the optical radiation pressure forces on the two interfaces (1-air/film interface, 2- film/air interface). The resultant forces are directed from the higher to the lower index medium. c) Picture of the film support that shows how the film is suspended.

$\nu_{l}$ is the optical frequency, and $\vec{k}$ is the wavevector $\left(k=2 \pi \nu_{l} / c, c\right.$ is the velocity of light in vacuum). $\vec{u}$ is a unitary vector collinear to $\vec{k}$. The term $(1-n)$ refers to the optical index discontinuity, $4 n /(n+1)^{2}$ accounts for the light intensity transmission. Since the force is associated with a change of linear momentum, it can thus be called an optical radiation pressure force [17].

This force has to be corrected for the force due to reflected light acting on this first interface $\vec{F}_{1}^{r}$ (see figure 1b). Since the momentum change upon reflexion is $2 \hbar \vec{k}$, this force is $F_{1}^{r}=((n-1) /(n+1))^{2} 2 P / c$. Finally, the force on the 
air/film interface is $F_{A / F}=F_{1}^{t}-F_{1}^{r}=7.0 \times 10^{-10} \mathrm{~N}$ for a $500 \mathrm{~mW}$ laser. For the second film/air interface, one has to consider the transmitted light from the air/film interface. The force due to the optical index variation is $\vec{F}_{2}^{t}=-\vec{F}_{1}^{t} \times 4 n /(n+1)^{2}$ and the force due to the reflected light is ${\overrightarrow{F_{2}}}^{r}={\overrightarrow{F_{1}}}^{r} \times 4 n^{2} /(n+1)^{2}$ acts on the same direction (see figure $1 \mathrm{~b}$ ) leading to a total force equal to $F_{F / A}=F_{2}^{t}+F_{2}^{r}=$ $1.02 \times 10^{-9} \mathrm{~N}$.

On each interface, the force is thus of the order of $F=1 \times 10^{-9} \mathrm{~N}$ and is always directed from the film to the air. The waist of the laser is $w_{w}=0.6 \mathrm{~mm}$, the value of the optical radiation pressure is then $p=1.4 \times 10^{-3} \mathrm{~Pa}$. When applied in a continuous way, this value is too low to induce any noticeable thickness variation due to stretching or surface bending linked to the stiffness properties of the sample. For example, considering the Young's modulus $E$, for a $e=220 \mu \mathrm{m}$ thickness, the thickness variation is $\Delta e=p / E \times e=1.5 \times 10^{-16} \mathrm{~m}$, which is hardly noticeable. The difficulty to deform an interface with the light radiation pressure is not limited to solids. This is also true for liquids. However, in this later case, in order to bend the interface, the light radiation pressure has to compensate for the surface tension of the liquid [18]. Unlike acoustic radiation pressure, the optical force is applied on a much smaller area of the sample allowing its interfacial properties to be probed locally.

\section{RESULTS}

To increase the surface deformation, as for the bending of air/liquid interfaces [7], we use the resonance of the sample under modulated optical radiation pressure force. We chop the laser beam at a given controlled frequency $\nu$. We measure the mean variation thickness of the film using the interference fringes from two He-Ne collimated laser probe beams: a red one $\left(\lambda_{R}=633 \mathrm{~nm}\right.$, beam waist $\left.w_{r}=400 \mu \mathrm{m}\right)$ and a green one $\left(\lambda_{G}=543 \mathrm{~nm}\right.$, beam waist $\left.w_{g}=380 \mu \mathrm{m}\right)[19]$ (see figure 1a). 


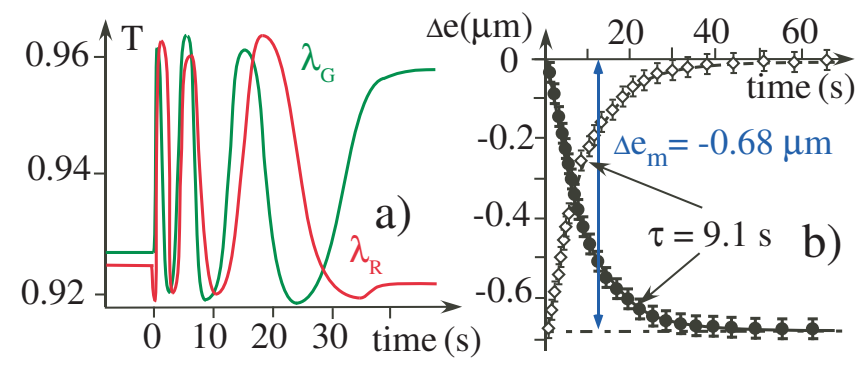

FIG. 2. a) T: intensity transmission recorded on the two photodiodes $\left(\mathrm{PD}_{\mathrm{R}}\right.$ and $\mathrm{PD}_{\mathrm{G}}$ versus time, at a frequency of $65.7 \mathrm{~Hz}$ and for a laser power of $500 \mathrm{~mW}$ ), when the laser is switched on; frequency sampling: $0.2 \mathrm{~Hz}$. b) Circles and solid line: variation of the film thickness versus time deduced from the interference fringes from a). Diamonds and doted line: variation of the film thickness when the laser is switched off. The error bars, obtained from at least 5 measurements at the same frequency, appear on the graph.

Both beams are partly reflected on each side of the film, leading to constructive or destructive interferences depending on the film thickness. As the thickness varies, the transmission varies accordingly, leading to fringes. A variation of one fringe then corresponds to a thickness variation of $\lambda / 2 n$.

An example of the transmission of the two laser beams versus time (sampling time: $0.01 \mathrm{~s}$ ) is displayed in figure $2 \mathrm{a}$, for a $500 \mathrm{~mW}$ laser beam at a modulation frequency of $65.7 \mathrm{~Hz}$. The two probe lasers are on. We switch on the modulated laser at time $t=0$ and we register the transmission of the probe beams versus time. The frequency has been chosen to optimize the number of fringes. A variation of a single fringe in the laser transmission in figure 2a corresponds to a thickness variation of half a wavelength $(\lambda /(2 n))$. There is thus a direct correspondence between the variation of the transmission and the variation of the thickness. We can follow the thickness variation of the film, which is then confirmed by the transmission of the second laser (see Fig. 2b). Note that the variation has to be higher than $\lambda / 2 n$ in order to register at least one fringe and thus to calibrate our 
observations.

The film experiences a succession of swelling and deflation leading, as for the liquid film [7], to a mean thinning. Usually, optical radiation pressure induces bulges on the film interfaces but we here measured dimples (see Fig. 2b). Even at resonance, and whatever the direction of the applied force, one should observe a mean response equal to zero, i.e. no variation in thickness. One has to dissociate (i) the vibrations of the system with forced oscillations, (ii) the maximum amplitude of the oscillations, and (iii) the mean thinning of the system induced by the vibrations. This thinning phenomenon may be attributed to the effects of negative compressibility of the material [20] that also appears in polymers [21]. This negative compressibility could be associated to rearrangements of the polymer chains due to resonant forced oscillations of the system. Then, within the stressed film, thinning is more effective than swelling, giving an asymmetric mechanical response.

The deformation equals $\Delta e=-680 \mathrm{~nm}$. It is worth noting that it corresponds to a static deformation of the film due to the modulated radiation pressure. The time constant associated with this deformation is $\tau=9.1 \mathrm{~s}$ (exponential adjustment). The amplitude of the oscillations was measured thanks to a lock-in amplifier. It corresponds to approximately $2 \mathrm{~nm}$ for a $500 \mathrm{~mW}$ excitation at the resonant frequency. The amplitude of the oscillation follows the same resonance curve as the mean deformation. For a power of $600 \mathrm{~mW}$, the thinning saturates to this same value. We have also performed experiments on a $20 \mu \mathrm{m}$ PVC film but didn't succeed in measuring thickness variations. Actually, assuming that the physical properties of PVC and Topas materials are similar and that the thinning of the sample is correlated with the sample thickness, a $60 \mathrm{~nm}$ thickness variation would be expected for the PVC. This value is well below $\lambda / 2 n$, and is then very difficult to measure.

We repeat the measurement for different frequencies and different optical pow- 
ers, and register the transmission of the green and red lasers. We apply the modulated laser for 2 minutes in order to reach a steady state, and switch off the laser for another 2 minutes to let the film relax before changing the frequency. The relaxation time constant is also equal to $9.1 \mathrm{~s}$ (see Fig. 2b). It is also the same for each laser power. Such transmissions versus the modulation frequency appear in figure $3 \mathrm{a}$ for a laser power of $300 \mathrm{~mW}$. Note that the number of fringes is higher for $500 \mathrm{~mW}$, but the results are less demonstrative. For the sake of simplicity, we present them for a $300 \mathrm{~mW}$ laser. From the transmission curves, we deduce the mean thickness variation versus the modulation frequency (see figure $3 \mathrm{~b}$ ). One clearly notices a resonant behavior at a frequency of $\nu=65.7 \mathrm{~Hz}$. The quality factor equals $Q=4$, assuming that the amplitude of the vibration is proportional to the mean thickness variation. Around resonance, the time constant $\tau$ associated to the thinning is unchanged. Far for resonance, it is more difficult to evaluate it. It is worth noting that the deformation is reversible since the polymer film recovers its initial shape after switching off the laser. Actually, the transmission of the probe lasers recovers its initial value.

This thickness variation must be linked to the resonance response of the sample under light radiation pressure. It cannot be attributed to a change of conformation due to the light irradiance [22] nor to a local heating because the light flux is the constant whatever the modulation frequency. Besides, Topas is transparent at these wavelengths and absorption is negligible. Since light is nothing but electromagnetic fields, one may wonder whether electrostriction could be responsible for this effect. However, electroactive polymer have been widely studied [23] and resonant responses under modulated excitation have never been reported.

We have varied the optical power of the modulated light (see figure 4a) and found the same resonant behavior. From a theoretical adjustment of the experimental data, we find the same resonant frequency and the same $Q$ factor for all the optical powers. One also notices that the mean thinning varies linearly with 

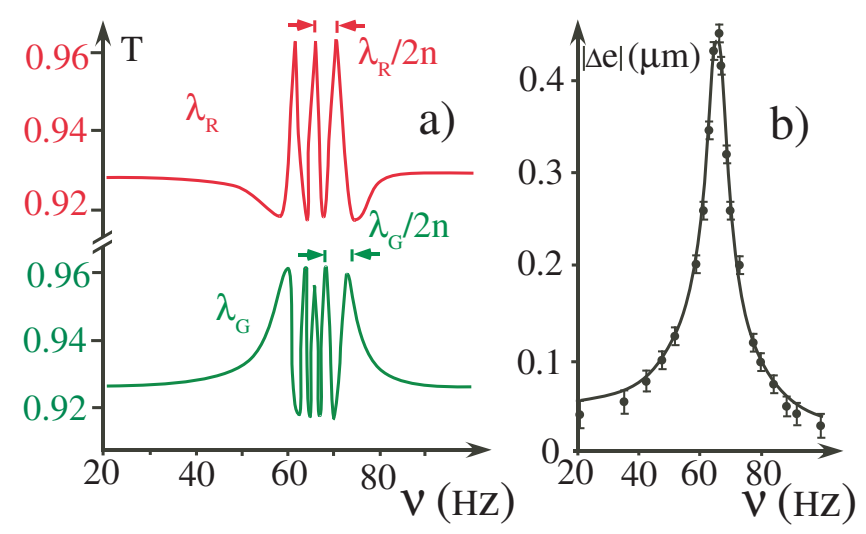

FIG. 3. a) Interference fringes of the transmissions $\mathrm{T}$ of the red and green laser versus modulation frequency, for a $\mathrm{P}=300 \mathrm{~mW}$ laser power, frequency sampling: $0.2 \mathrm{~Hz}$. b) From the fringes, one can deduce the absolute value of the thickness variation versus modulation frequency. The solid line corresponds to a fit of the experimental curve using equation 3. Error bars: same as Fig. 2.

the optical power as expected (see figure 4b) up to a power a $500 \mathrm{~mW}$. We were not able to make a complete cartography of the surface of the film because the amplitudes, out of the irradiated surface must be too low.

\section{DISCUSSION}

\section{Optically induced mechanical oscillations}

What could be the origin of such a resonance that resembles the mechanical oscillator behavior? Let us consider the equation of the weakly damped mechanical oscillator excited at a pulsation $\omega$ applied to our experiment

$$
m \ddot{x}+\alpha \dot{x}+k_{s} x=F_{A / F} \cos (\omega t)
$$

where we consider a single interface. Actually, since we excite the interface in a symmetric way (see section ), we thus expect the deformation to be symmetric, 
corresponding to a breathing mode where both interfaces oscillate in a symmetric way. This type of vibration is opposed to the usual bending mode where both interfaces oscillate in an antisymmetric way. We can then only consider half of the film.

$x$ corresponds to the amplitude of the forced oscillation around a zero position. It is in the direction of the force. $m$ is the mass of the film, $\alpha$ is the viscous damping coefficient, $k_{s}$ is a spring constant, and $\omega$ is the driving pulsation. Note that the laser is modulated with a chopper leading to a square modulation of the light intensity. However, we don't observe experimentally any response at twice, and three times the $\omega$ pulsation, either on the mean thinning or on the amplitude of the oscillation. We could then consider a cosine modulation of the laser only. Note also that Eq. 2 corresponds to a forcing with positive and negative values whereas the optical radiation pressure force here always acts outward. Nevertheless, we can add a constant force in Eq. 2 that equals half of the value of the optical radiation pressure force term. Thus, the remaining part of the force would be a forcing term with positive and negative values. This constant term could then be moved to the other side of the equation and would behave as an offset (or a change of origin in the vibration amplitude $x$ ). Let us introduce the natural resonant pulsation $\omega_{0}=\sqrt{k_{s} / m}$. The well-known solution of equation 2 is $x(t)=x_{A} \cos (\omega t+\phi), \phi$ being the phase between the excitation and the response, and

$$
x_{A}=\frac{F_{A / F}}{m} \frac{1}{\sqrt{\left(\omega^{2}-\omega_{0}^{2}\right)^{2}+\alpha^{2} \omega^{2} / m^{2}}}
$$

The resonant pulsation $\omega_{R}$ in position equals

$$
\omega_{R}=\omega_{0}\left(1-\frac{1}{2 Q^{2}}\right)^{1 / 2}
$$

Since the experimental resonant pulsation is $2 \pi \times 65.7 \mathrm{rad} \times \mathrm{s}^{-1}$, the natural resonant pulsation is equal to $\omega_{0}=2 \pi \times 66.7 \mathrm{rad} \times \mathrm{s}^{-1}$, according to equation 4 . It is worth noting that in the case of capillary waves in ultra thin liquid films 


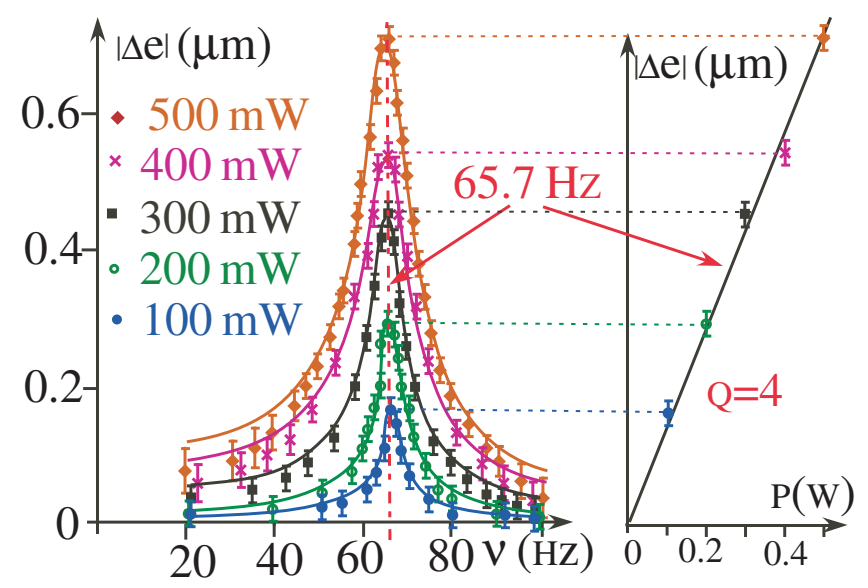

FIG. 4. Absolute value of the thinning versus the modulation frequency for various laser powers (the solid lines correspond to theoretical fits), together with the maximum thickness variation versus power at the resonant frequency $\nu=65.7 \mathrm{~Hz}$. Straight line: theoretical adjustment according to Eq. 3. Error bars: same as Fig. 2.

(thickness of the order of $100 \mathrm{~nm}$ ), the resonant frequency depends on the film thickness [24]. However, since the thickness in our experiment is $220 \mu \mathrm{m}$, we can neglect this dependency and can consider the resonant frequency as a constant.

Let us comment briefly on some of the terms that appear in the simple model of Eq. 2. Whereas the force is applied on a very specific zone of the surface of the sample, its response in our model includes its whole mass and not only the mass underneath the laser. In order to check this hypothesis, we have cut our sample in two pieces with the same thickness $60 \times 21 \mathrm{~mm}^{2}$, total mass $m^{\prime}=282 \mathrm{mg}$ ), and we have looked for a resonance. The new natural resonant pulsation should be $\omega_{0}^{\prime}=2 \pi \times 93.6 \mathrm{rad} \times \mathrm{s}^{-1}$, and the quality factor $Q^{\prime}=2$ according to Eq. 4. The new resonant pulsation should be $\omega_{R}^{\prime}=2 \pi \times 87.5 \mathrm{rad} \times \mathrm{s}^{-1}$.

The resonance curve versus frequency for $m^{\prime}$ appears in Fig. 5, together with the resonance curve for $m$ taken from Fig. 4, for a laser power of $500 \mathrm{~mW}$. Experimentally we find $\omega_{R}^{\prime}=2 \pi \times 84.6 \mathrm{rad} \times \mathrm{s}^{-1}$ that is very close to the expected value. The amplitude of the resonance is diminished, and $Q^{\prime}=2$, as expected 


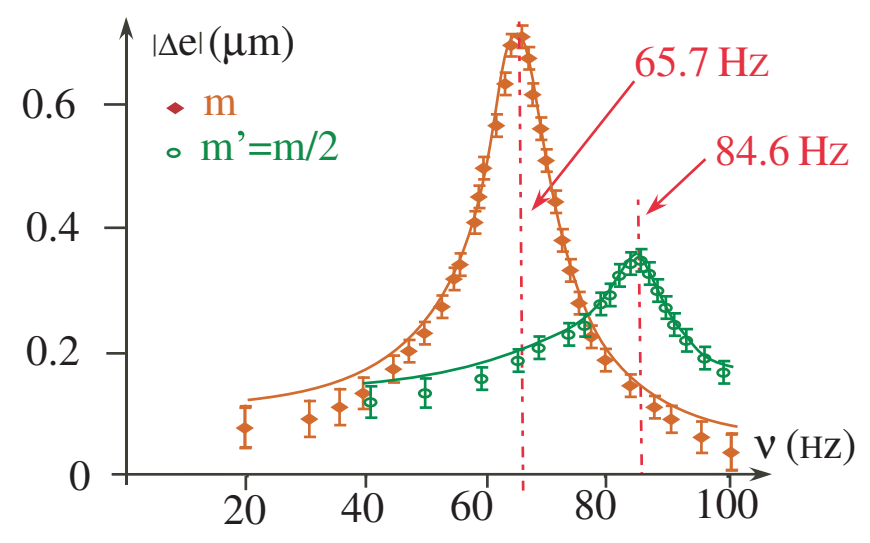

FIG. 5. Absolute value of the thinning for two different masses $\left(m=565 \mathrm{mg}, m^{\prime}=285\right.$ $\mathrm{mg}$ ) of the sample versus the modulation frequency. Straight line: theoretical adjustment. Error bars: same as Fig. 2. Resonant frequency $\nu_{R}=65.7 \mathrm{~Hz}$ for $m$, and $\nu_{R}^{\prime}=84.6 \mathrm{~Hz}$ for $m^{\prime}$.

from Eqs. 3 and 4. The fitting parameters are also in agreement with Eq. 3.

By analogy with our experiments on air/liquid interfaces [7], we attribute this resonance phenomenon to the surface tension contribution of the film. The sample is too thick to take into account elastic bending [25] and compressibility effects. By analogy with the tensiometer [26], the spring constant should be proportional to the surface tension $\gamma$ times the contour on which the tension is applied, divided to the maximum elongation which is $\Delta e / 2$ for one interface. It can be written as $k_{s}=2 \pi w_{w} \gamma /(\Delta e / 2)$. Let us recall that $w_{w}$ is the beam waist. Then the natural resonant pulsation is

$$
\omega_{0}=\sqrt{\frac{4 \pi w_{w} \gamma}{m|\Delta e|}}
$$

For $m=5.65 \times 10^{-4} \mathrm{~kg}, \Delta e=-680 \mathrm{~nm}$, and for an optical power of $500 \mathrm{~mW}$ and beyond, this leads to $\gamma=1.0 \times 10^{-2} \mathrm{~N} \times \mathrm{m}^{-1}$ which is of the same order of the value of $\gamma=3.2 \times 10^{-2} \mathrm{~N} \times \mathrm{m}^{-1}$ measured indirectly using contact-angle techniques [27], although the measurement was performed with a different Topas grade.

One can argue that this resonance may be due to the bulk elastic proper- 
ties of the polymer film. Then the natural resonant pulsation equals $\omega_{0}=$ $A \sqrt{E e^{2} /\left(\rho\left(2 \pi w_{w}\right)^{4}\left(1-\sigma^{2}\right)\right)}$ where the calculated constant $A$ depends on the film geometry [28] (here $A=6.09 \mathrm{rad} \times \mathrm{s}^{-1}$ ). For our experimental parameters, the $\omega_{0}$ value would be $\omega_{0}=2 \pi \times 150 \times 10^{3} \mathrm{rad} \times \mathrm{s}^{-1}$, i.e. between $100 \mathrm{kHz}$ and 1 $\mathrm{MHz}$. This is very different from the experimental values we found. Besides, as already mentioned, the stress imposed by the laser light would correspond to a pressure below the $\mathrm{mPa}$ range and a deformation of the sample that is very low (in the $10^{-16} \mathrm{~m}$ range) if it was only due to the elastic properties of the film. Even with a sharp and strong resonance, this elastic effect should not be noticeable. We can thus neglect the mechanical properties of the film in volume and concentrate on the deformation due to the interfacial properties only.

One may nevertheless express some reservations about the one-dimensional model (Eq.3), since the Gaussian laser beam acts on a surface perpendicular to the thickness. At least a two-dimensional description could be needed, including the radial intensity distribution of the laser beam. Although this description is far beyond the scope of the article, one may note that our one-dimensional model gives the correct orders of magnitude. Indeed, the capillary length, $\lambda_{c}$, is used to estimate the size of the deformed surface. Knowing that $\lambda_{c}=(\gamma /(\rho g))^{1 / 2}$, where $g$ is the gravitational acceleration, one finds that $\lambda_{c}$ is approximately equivalent to the laser waist (about $1 \mathrm{~mm}$ ). We could thus in first approximation forget the radial distribution of the laser intensity and also of the deformation of the film surface.

\section{Energy storage}

From equation 3, one can deduce the $Q$ factor of the resonance $Q=m \omega_{0} / \alpha$, and thus, from the value derived from figure 3 , we estimate the damping coefficient $\alpha=$ $6.23 \times 10^{-2} \mathrm{~kg} \times \mathrm{s}^{-1}$. It has also to be noted that the time constant deduced from this 
equation $m / \alpha=Q / \omega_{0}=9.07 \times 10^{-3} \mathrm{~s}$ is very different from the value of $\tau=9.1 \mathrm{~s}$ deduced from figure 2. Actually, they do not correspond to the same phenomenon. $m / \alpha$ is the time constant associated with a single excitation of the sample, whereas $\tau$ is the time associated with the mean thinning of the sample while periodically excited. It is worth noting that the value of $\tau=9.1 \mathrm{~s}$ is a rather long time. One hypothesis is that the relaxation time of the polymer chains, especially at the interface, is much longer than the elastic relaxation time of the system. Its characteristic time could be much higher than the one-second range [29]. It is difficult to compare the relaxation time they found with what we measured since they used a photobleaching technics that depends on the fluorescence probes.

Besides, since this coefficient $\alpha$ corresponds to an energy damping, it should give access to the elastic properties of the sample. In particular, the imaginary part of the Young's modulus $E^{\prime \prime}$ is related to the energy dissipation. One can define $\alpha=E^{\prime \prime}\left(2 \pi w_{w} e / V\right)$, where $2 \pi w_{w} e$ is the surface of the cylinder underneath the region enlightened by the laser, and $V=\sqrt{E /(2 \rho(1+\sigma))}=913 \mathrm{~m} \times \mathrm{s}^{-1}$ is the sound velocity in the medium. This then leads to $E^{\prime \prime}=74 \mathrm{MPa}$ and to a loss factor $\tan \delta=E^{\prime \prime} / E \simeq 0.03$ that is compatible with the values deduced from Dynamical Mechanical Analysis (DMA) [30]. The damping coefficient could then be associated with the imaginary part of the elastic mechanical coefficient of the sample. However, whereas for a usual damped mechanical oscillator, the energy is dissipated into heat, here, part of the energy is stored within the material and then restored to the system that recovers its initial shape.

This stored energy can be estimated in two ways. First, the power lost by a mechanical damped harmonic oscillator is $(1 / 2) \alpha \dot{x}_{A}^{2}$, where $\dot{x}_{A}$ is the oscillation velocity amplitude. At resonance, on the first interface $\dot{x}_{A}=F_{A / F} / \alpha$, according to equation 3. During a time $\tau$, the energy dissipation is then $\tau F_{A / F}^{2} / 2 \alpha=3.6 \times 10^{-17}$ $\mathrm{J}$, for the first interface and $7.6 \times 10^{-17} \mathrm{~J}$ for the second interface. There is also a mean variation $\Delta e$ of the thickness during this time. The corresponding velocity 
is $(\Delta e) / \tau$, associated with a damping force $\alpha(\Delta e) / \tau$ on a distance $(\Delta e)$, leading to a lost $\left(\alpha(\Delta e)^{2}\right) / \tau=3.1 \times 10^{-15} \mathrm{~J}$. The total energy lost is of the order of $\Delta E=3.3 \times 10^{-15} \mathrm{~J}$. The mean thinning when light is on, as the one shown in figure $3 \mathrm{~b}$, corresponds to an energy storage in the film.

The stored energy can also be estimated using a thermodynamic approach and considering the free energy of the system. The temperature is constant (see experimental conditions described in figure 1a). The surface deformation on each interface equals $\Delta S=\pi / 2(\Delta e / 2)^{2}$, assuming a deformation following the Gaussian laser intensity distribution. The same results hold for a conical deformation, and the order of magnitude is the same whatever the deformation shape. Then, for the two interfaces, the free energy variation is $\Delta F=(\pi / 4) \Delta e^{2} \gamma$. Considering the value of $\gamma=1.0 \times 10^{-2} \mathrm{~N} \times \mathrm{m}^{-1}$ that has been already estimated, the variation of the free energy is $3.6 \times 10^{-15} \mathrm{~J}$. It is of the same order of magnitude as the energy lost during the time $\tau$. This reinforces the hypothesis that the deformation is due to the interfacial properties of the polymer film.

\section{CONCLUSION}

To conclude, we have experimentally shown a resonant breathing mode of a 220 $\mu \mathrm{m}$ thick thermoplastic film. The excitation is performed by the radiation pressure of a visible laser that leads to an antisymmetric vibration on both interfaces of the film. The thinning of the film is a fraction of a micrometer, i.e. in the 0.1 percent range of the initial thickness. However, it could be detrimental when the thermoplastic is used as an optical material. For example, in some optogenetic interrogation experiment, light is guided by polymer fibers [31]. Modulating the light intensity may lead to the deformation of the fiber surface and consequently to the change of the light injection, that thus may change the diagnosis or the analysis. Alternatively, such resonant phenomena could initiate the bending or the twisting 
in multilayers thermoplastic polymers [32] or favor the return to equilibrium of shape memory polymers [33].

This thickness variation is reversible. It disappears as the laser excitation is switched off. Besides, the effect is a local effect. The response of the film is governed by its surface tension, deduced from the experimental resonant frequency. Using a spring model, the damping of the oscillations is linked to the imaginary part of the Young's modulus. These experimental results show that the surface tension of a solid material can be measured directly, without using contact angle methods with a series of liquids. Besides, the technique developed here is not limited to thermoplastic polymers and may be generalized to measure the surface tension of solids in general whose thermodynamic definition is confusing compared to liquids $[34,35]$, and where reliable experimental data are scarce [36, 37].

* Corresponding author; olivier.emile@univ-rennes1.fr

[1] K. Ogata, System dynamics, 7 (Prentice-Hall Englewood Cliffs, NJ, 2004).

[2] D. Halliday, R. Resnick, and J. Walker, Fundamentals of physics (John Wiley \& Sons, 2013).

[3] C. H. Jenkins and U. A. Korde, J. Sound Vib. 295, 602 (2006).

[4] J. P. Fay, S. Puria, and C. R. Steele, Proc. Natl. Acad. Sci. 103, 19743 (2006).

[5] K. Nan, H. Wang, X. Ning, K. A. Miller, C. Wei, Y. Liu, H. Li, Y. Xue, Z. Xie, H. Luan, Y. Zhang, Y. Huang, J. A. Rogers, and P. V. Braun, ACS Nano 13, 449 (2018).

[6] A. Boudaoud, Y. Couder, and M. B. Amar, Phys. Rev. Lett. 82, 3847 (1999).

[7] O. Emile and J. Emile, Lab Chip 14, 3525 (2014).

[8] O. Emile and J. Emile, Optofluid. Microfluid. Nanofluid. 3, 49 (2016).

[9] J. Emile, Y. Gueguen, J.-C. Sangleboeuf, V. Nazabal, and O. Emile, J. Non-Cryst. Solids 535, 119962 (2020). 
[10] D. A. Mair, E. Geiger, A. P. Pisano, J. M. Fréchet, and F. Svec, Lab Chip 6, 1346 (2006).

[11] E. Roy, J.-C. Galas, and T. Veres, Lab Chip 11, 3193 (2011).

[12] P. N. Nge, C. I. Rogers, and A. T. Woolley, Chem. Rev. 113, 2550 (2013).

$[13]$.

[14] T. Nielsen, D. Nilsson, F. Bundgaard, P. Shi, P. Szabo, O. Geschke, and A. Kristensen, J. Vac. Sci. Technol. B 22, 1770 (2004).

[15] R. W. Style, R. Boltyanskiy, Y. Che, J. Wettlaufer, L. A. Wilen, and E. R. Dufresne, Phys. Rev. Lett. 110, 066103 (2013).

[16] A. Ashkin and J. M. Dziedzic, Phys. Rev. Lett. 30, 139 (1973).

[17] O. Emile and J. Emile, Ann. Phys. 530, 1800111 (2018).

[18] A. Casner and J.-P. Delville, Phys. Rev. Lett. 87, 054503 (2001).

[19] J. Emile, F. Casanova, and O. Emile, Soft Matter 8, 7223 (2012).

[20] A. B. Cairns and A. L. Goodwin, Physical Chemistry Chemical Physics 17, 20449 (2015).

[21] W. Miller, K. E. Evans, and A. Marmier, Appl. Phys. Lett. 106, 231903 (2015).

[22] M. R. Aguilar and J. San Román, Smart Polymers and Their Applications (Elsevier, Cambridge, 2019).

[23] Y. Bar-Cohen and I. A. Anderson, Mech. Soft Mater. 1, 5 (2019).

[24] M. L. Henle and A. J. Levine, Phys. Rev. E 75, 021604 (2007).

[25] J. Bae, T. Ouchi, and R. C. Hayward, ACS Appl. Mater. Interfaces 7, 14734 (2015).

[26] H. J. Butt, K. Graf, and M. Kappl, Physics and chemistry of interfaces (John Wiley \& Sons, Weinheim, 2013).

[27] R. K. Jena, C. Y. Yue, Y. C. Lam, P. S. Tang, and A. Gupta, Sens. Actuators B Chem. 163, 233 (2012).

[28] C. M. Harris and A. G. Piersol, Harris' shock and vibration handbook, Vol. 5 (McGraw-Hill, New York, 2002). 
[29] K. Paeng, S. F. Swallen, and M. Ediger, J. Am. Chem. Soc. 133, 8444 (2011).

[30] K. P. Menard and N. Menard, Encyclopedia of Analytical Chemistry: Applications, Theory and Instrumentation (John Wiley \& Sons, Weinheim, 2006).

[31] S. Park, Y. Guo, X. Jia, H. K. Choe, B. Grena, J. Kang, J. Park, C. Lu, A. Canales, R. Chen, Y. S. Yim, G. B. Choi, Y. Fink, and P. Anikeeva, Nat. Neurosci. 20, 612 (2017).

[32] C. E. Wisinger, L. A. Maynard, and J. R. Barone, Soft Matter 15, 4541 (2019).

[33] E. Kurahashi, H. Sugimoto, E. Nakanishi, K. Nagata, and K. Inomata, Soft Matter 8, 496 (2012).

[34] L. Makkonen, Langmuir 30, 2580 (2014).

[35] P. Lazar and M. Otyepka, Phys. Rev. B 91, 115402 (2015).

[36] A. Zdziennicka, K. Szymczyk, J. Krawczyk, and B. Jańczuk, Appl. Surf. Sci. 405, 88 (2017).

[37] Z. Chen and M. Nosonovsky, Surf. Topogr.: Metrol. Prop. 5, 045001 (2017). 